\title{
An Apparently Balanced Complex Chromosome Rearrangement Involving Seven Breaks and Four Chromosomes in a Healthy Female and Segregation/Recombination in Her Affected Son
}

\author{
Ana Eduarda Campos ${ }^{a}$ Carla Rosenberg ${ }^{b, d}$ Ana Krepischi ${ }^{d}$ Marina França ${ }^{a}$ \\ Vanessa Lopes $^{b}$ Viviane Nakano $^{b}$ Tânia Vertematic Marcos Cochak $^{b}$ Michele Migliavaccab \\ Fernanda Milanezi $^{b} \quad$ Ana Cristina Sousa ${ }^{b}$ Juliana Silva ${ }^{b}$ Lígia Vieira $^{b}$ Priscilla Monfredini $^{b}$ \\ Ana Carolina Palumbo ${ }^{b}$ Jonathas Fernandes ${ }^{b}$ Eduardo Perrone ${ }^{a, b}$ \\ aDepartment of Clinical Genetics, Federal University of São Paulo, São Paulo, Brazil; 'beneOne, São Paulo, \\ Brazil; ' Private Genetics Clinic, São Paulo, Brazil; ${ }^{d}$ Department of Genetics and Evolutionary Biology, Institute of \\ Biosciences, Human Genome and Stem Cell Research Center, University of São Paulo, São Paulo, Brazil
}

\section{Established Facts}

- Constitutional complex chromosomal rearrangements (CCRs) are rare and may arise through replication, repair or recombination errors.

- CCRs can result in normal or abnormal phenotypes, depending on whether the respective genome is balanced, and on the changes caused by the breakpoints on coding or regulatory elements.

- Affected or unviable offspring typically result from different combinations of the derivative and nonrearranged chromosomes due to segregation in the gametes. More rarely, meiotic recombination between the normal and rearranged homologues may result in new unbalances in the offspring.

- The frequency of CCR in the general population may be underestimated; when balanced, they may not have a phenotypic effect.

\section{Novel Insights}

- To the best of our knowledge, no other constitutional balanced CCR involving chromosomes 1, 4, 14, and 18 has been previously described.

- The proband inherited 3 derivative chromosomes, and 1 chromosome originated from the recombination between the normal homologue and a derivative chromosome, resulting in 2 duplications.

- Thus, our patient exhibits an exceptional clinical picture, demonstrating key features of 1q41q43 duplication and 4p15.2p14 duplication syndromes.

\section{Keywords}

Chromosome rearrangement · Karyotype $\cdot$ Duplication $4 p \cdot$ Duplication $1 \mathrm{q} \cdot \mathrm{FISH}$

\section{Abstract}

Duplication of the distal $1 q$ and $4 p$ segments are both characterized by the presence of intellectual disability/neurodevelopmental delay and dysmorphisms. Here, we describe a
Correspondence to:

Ana Eduarda Campos, anaepcampos@gmail.com 
male with a complex chromosome rearrangement (CCR) presenting with overlapping clinical findings between these 2 syndromes. In order to better characterize this CCR, classical karyotyping, FISH, and chromosomal microarray analysis were performed on material from the patient and his parents, which revealed an unbalanced karyotype with duplications at $1 \mathrm{q} 41 \mathrm{q} 43$ and $4 \mathrm{p} 15.2 \mathrm{p} 14$ in the proband. The rearrangements, which were derived from a maternal balanced karyotype, included an insertion of a segment from the long to the short arm of chromosome 1, a balanced translocation involving chromosomes 14 and 18 , and an insertion of a segment from the short arm of chromosome 4 into the derived chromosome 14. This study aimed to better define the clinical history and prognosis of a patient with this rare category of chromosomal aberration. Our results suggest that the frequency of CCR in the general population may be underestimated; when balanced, they may not have a phenotypic effect. Moreover, they emphasize the need for cytogenetic techniques complementary to chromosomal microarray for proper genetic counseling.

(c) 2021 S. Karger AG, Basel

\section{Introduction}

Chromosomal alterations are a major cause of morbidity and mortality in humans; a small fraction of them are complex chromosomal rearrangements (CCR), which are rare structural alterations [Berend et al., 2002; Scriven et al., 2014] involving 3 or more chromosomes. Most of them are considered unique, as they are found only in members of the same family [Shaffer and Lupski, 2000; Patsalis et al., 2004; Ciccone et al., 2005], leading to dis- tinct reproductive risks for balanced carriers with normal phenotypes. These carriers often only discover their status after the birth of an affected child, whose phenotype depends on the size and genomic content of the imbalances [Neira et al., 2012; Rivera and Dominguez, 2012]. Most carriers are women [Batista et al., 1994; Zhang et al., 2009], diagnosed after recurrent miscarriages, a history of infertility, or, as mentioned, the birth of an affected child. According to Liao et al. [2017], the general risk for spontaneous abortions for CCR carriers is $77.6 \%$, and $9.7 \%$ for an affected child. Most unbalanced CCRs occur as de novo events and are often associated with intellectual disability.

Segregation during meiosis in CCR carriers is the main driver of structural alterations, generating unbalanced gametes containing random combinations of derivative and normal homologues; the probability of producing gametes with balanced genomes decreases as the number of chromosomes involved in the rearrangements increases. It is rare to find children carrying new derivative chromosomes originating from crossingover between normal and rearranged homologues from balanced CCR carriers [Madan, 2012]. As the occurrence of meiotic recombination in some CCRs is an established fact, the pairing between these partially homologous chromosomes may be compromised [Kurtas et al., 2019].

In recent decades, several studies have used chromosomal microarray techniques to reveal cryptic rearrangements. There is a direct relationship between the number of breakpoints detected and the resolution of the analysis method used to assess CCRs because when the resolution increases, the identified number of breakpoints also tends
Fig. 1. a, b Dysmorphic facial features of the patient at the age of 2 years. Note microcephaly, triangular face, discrete synophrys, simplified ear shape, prominent nose, thin upper lip, and slight retrognathia. a

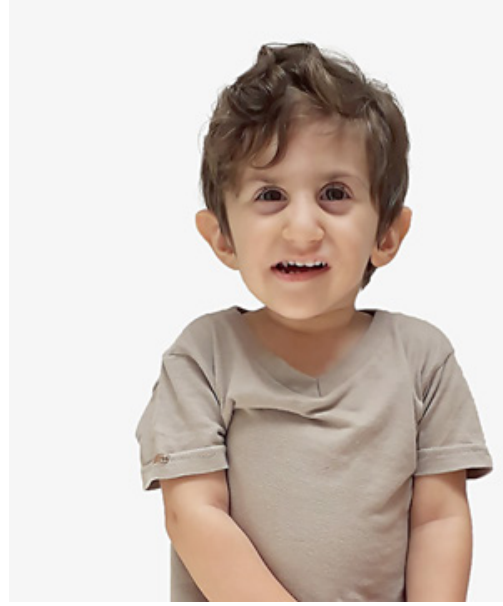

b

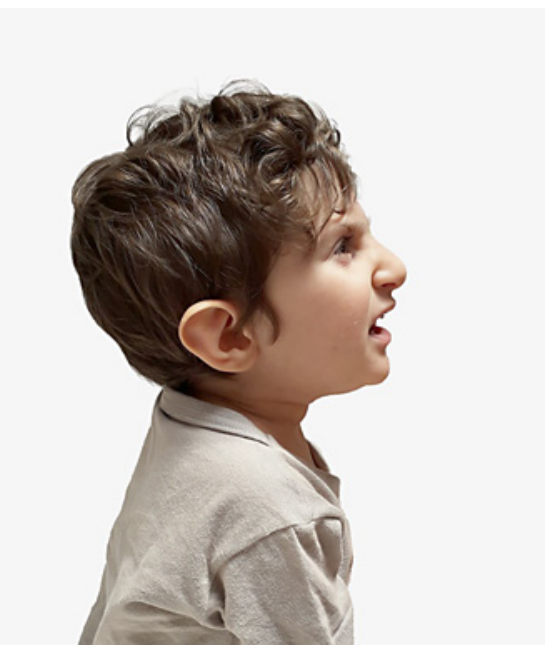

Segregation and Recombination of a Balanced 7 Breakpoint CCR
Mol Syndromol 2021;12:312-320 DOI: $10.1159 / 000516323$ 
a

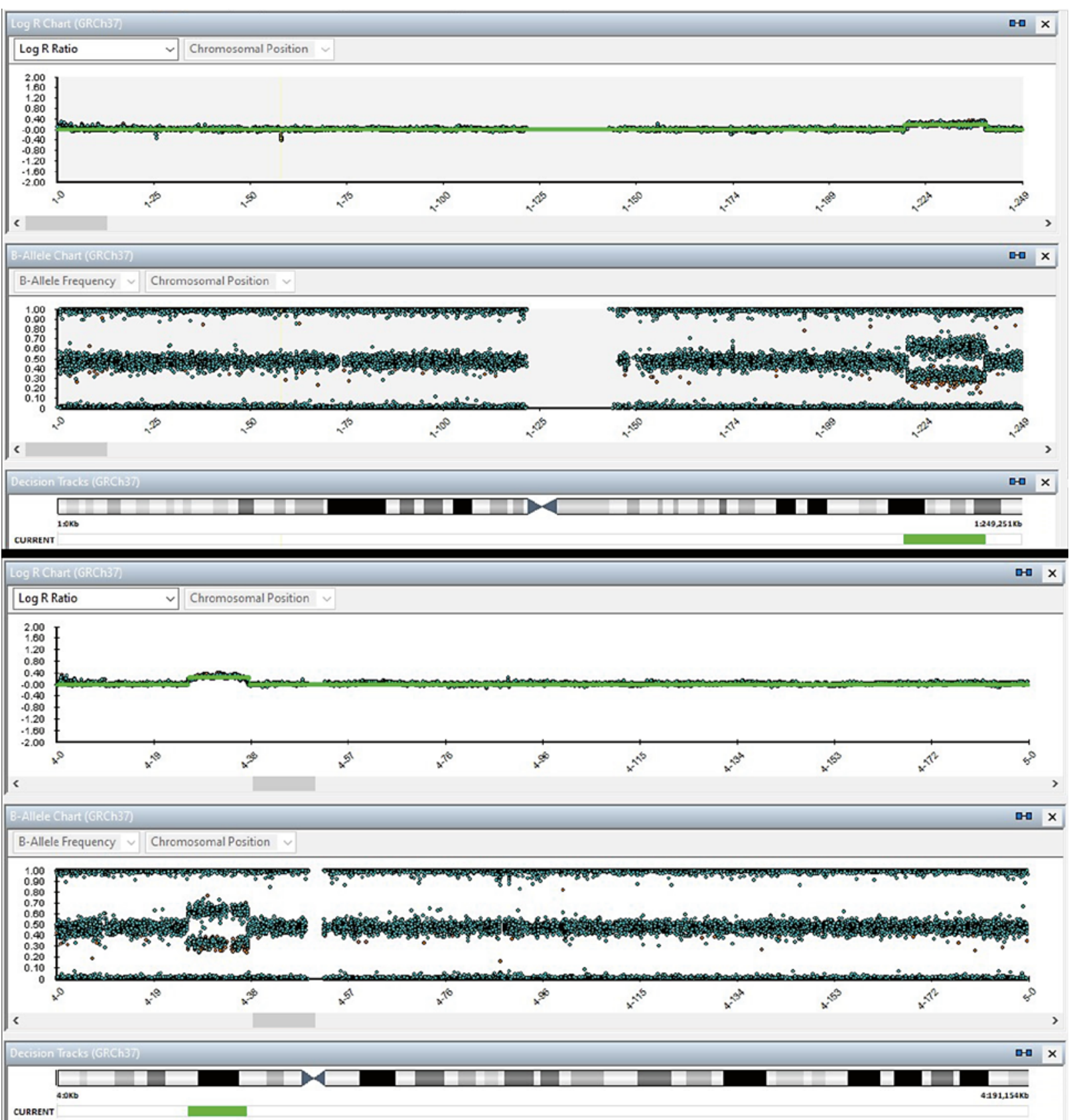

Fig. 2. Proband's chromosome microarray analyses. a Segment 1q. b Segment 4p.

to increase [De Gregori et al., 2007; Sismani et al., 2008; Poot and Haaf, 2015]. This observation suggests that many, and possibly the majority, of CCRs detected to date might actually be more complex than initially thought.

Although chromosome microarray analysis (CMA) has improved our ability to detect and characterize un- balanced CCRs, balanced structural chromosome abnormalities are not detected by this technique and require the use of additional classical cytogenetic techniques for more accurate characterization of the rearrangements and proper genetic counseling [Nazaryan et al., 2014]. 


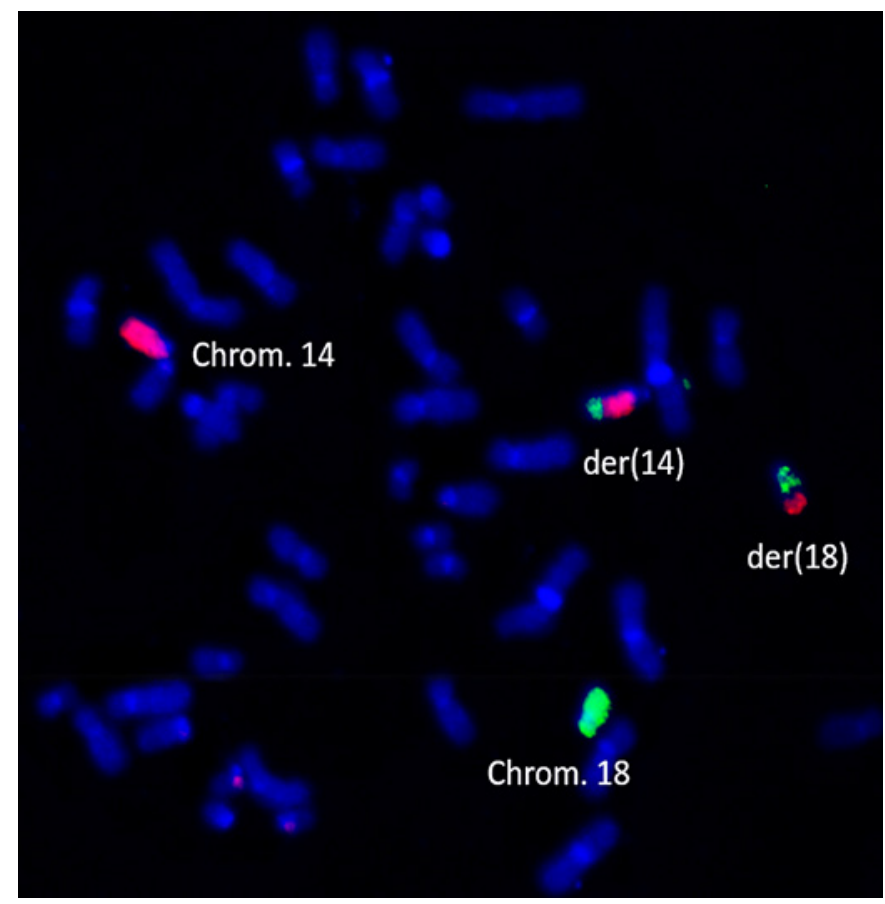

Fig. 3. FISH with libraries for chromosome 14 (red) (RP11-815P21) and 18 (green) (RP11-87C15). Metaphases from the proband show an apparently reciprocal translocation between these chromosomes inherited from his mother.

Herein, we report on a male patient, who presented with phenotypic and neurodevelopmental abnormalities and inherited 3 derivative and 1 recombinant chromosome. We also describe findings of his mother, a phenotypically normal female with a balanced CCR involving 7 breaks and 4 chromosomes, presenting recurrent abortions. Three distinct molecular techniques (SNP array, FISH, and karyotyping) were necessary to determine the structure of the chromosome rearrangements and to clarify the clinical presentation of the proband (child).

\section{Case Report}

The proband, a 2-year-old male, was the product of the fourth pregnancy of a nonconsanguineous healthy couple following 2 miscarriages and the birth of a healthy girl. Ultrasound at 24 weeks' gestation showed intrauterine growth restriction. The patient was delivered by cesarean section at the 38th week; birth weight, length, and head circumference were $2,195 \mathrm{~g}$ ( $<3 \mathrm{rd}$ percentile), $46 \mathrm{~cm}$ (3rd-10th percentile), and $32 \mathrm{~cm}$ (3rd-10th percentile), respectively. At physical examination (2 years old), the child had developmental delay, notably in language; microcephaly; failure to thrive, and dysmorphic features (a triangular face, synophrys, simplified ear shape, a thin upper lip, slight retrognathia, a deep hallucal crease, overlapping 2 nd and $3 \mathrm{rd}$ toes, and flat hemangioma in the dorsal region) (Fig. 1). Complementary exams showed an interatrial septal defect (ostium secundum type) and a moderate right heart dilatation. He underwent surgical correction, with good outcomes. Brain MRI showed no abnormalities. An ophthalmological evaluation was also normal.

Genomic DNA samples from the blood of both the proband and his mother were hybridized, according to the manufacturer's instructions to a CytoSNP 850K BeadChip (Illumina, San Diego, CA, USA), containing 850,000 SNP probes covering the whole genome. Data were analyzed using BlueFuse Multi 4.5 Software (BlueGnome Ltd., Cambridge, UK), and log ratio and B-allele frequency values were plotted along the chromosomal coordinates.

Metaphase chromosome spread preparations were obtained from lymphocyte cultures of peripheral blood samples from the proband and his mother, and G-banded karyotyping was performed according to standard protocols. FISH was performed on chromosome preparations. BAC probes were chosen from the Wellcome Trust $1 \mathrm{Mb}$ clone set (http://feb2014.archive.ensembl.org/index. html) to either map at subtelomeric regions of chromosomes or within the regions found by CMA to be duplicated in the proband. BAC DNAs were labeled by nick translation, either with biotin (visualized with FITC) or digoxigenin (visualized with rhodamine) (online suppl. Table 3; for all online suppl. material, see www.karger. com/doi/10.1159/ 000516323).

The previous G-banding result of the proband was reported as normal 46,XY. CMA analysis of the proband, performed using the Illumina $850 \mathrm{k}$ platform, revealed duplications of $20.3 \mathrm{Mb}$ and $11.8 \mathrm{Mb}$ segments from $1 \mathrm{q}$ and 4p, respectively: arr[GRCh37] 1q41q43(219446253 $239755240) \times 3 ; \quad 4 p 15.2 p 14(25803830-37653656) \times 3$ (Fig. 2). Online supplementary Tables 1 and 2 list all OMIM genes comprised in the $1 \mathrm{q}$ and $4 \mathrm{p}$ duplications segments.

The mother's G-banded karyotype was $46, X X, t(1 ; 14 ; 18 ; 4)(q ? 41 ; q ? 24 ; q ? 21 ; p ? 15)$, and CMA showed no abnormalities.

FISH libraries for chromosomes 14 and 18 confirmed the $14 ; 18$ translocation in the proband (Fig. 3 ) inherited from his mother. FISH results showed that the duplicated segments from 1q41q43 and 4p15.2p14 were inserted in 

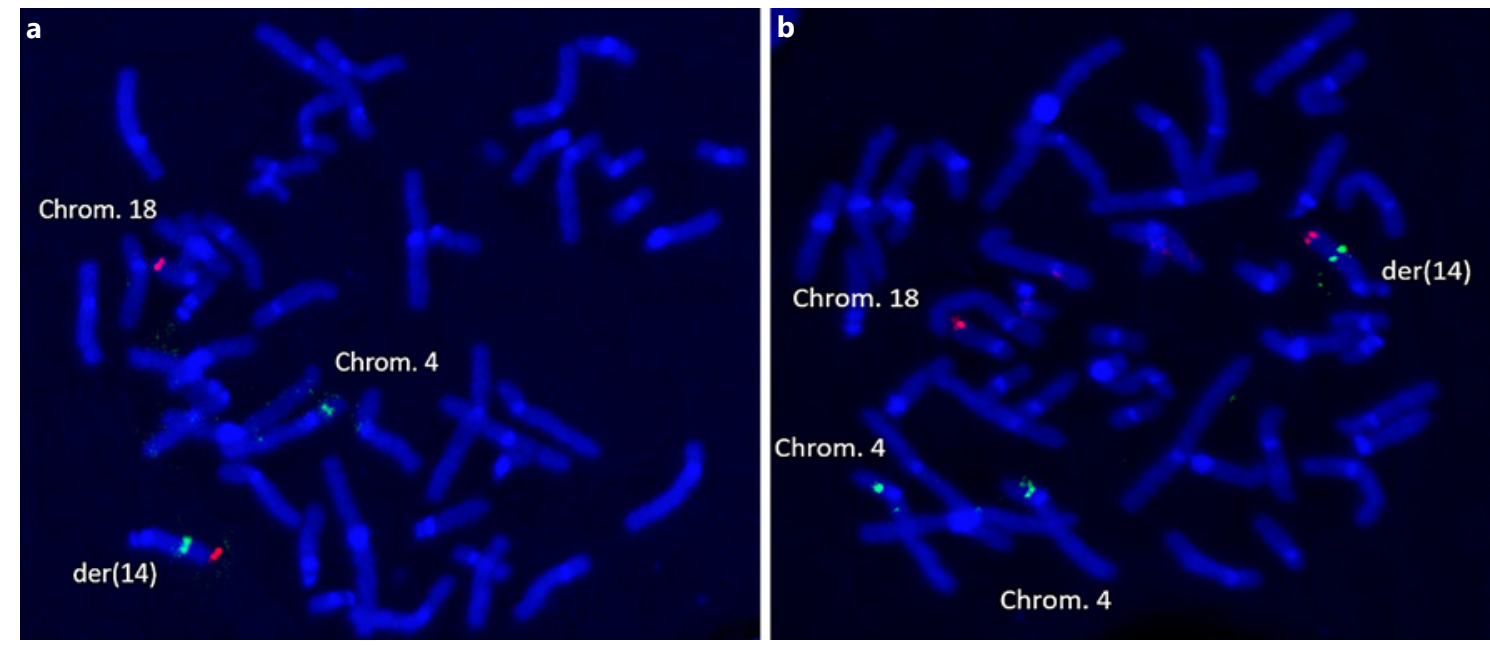

Fig. 4. FISH with an interstitial probe for the short arm of chromosome 4 (green) (RP11-390C19) and another that maps to 18qter (red) (RP11-87C15). a Metaphase from the mother showing a balanced rearrangement: only one chromosome 4 and one chromosome 18 exhibiting the expected signals, and the other signals from $4 \mathrm{p}$ and $18 \mathrm{q}$ mapping to the $\operatorname{der}(14)$. $\mathbf{b}$ Metaphase from the proband showing an unbalanced rearrangement: chromosome 18 shows two signals, one on chromosome 18 and one on $\operatorname{der}(14)$; however, there are three signals from chromosome 4, two normal chromosome 4 homologues plus one signal on the der(14). This result is in agreement with the duplication in $4 \mathrm{p}$ observed by chromosome microarray analyses.
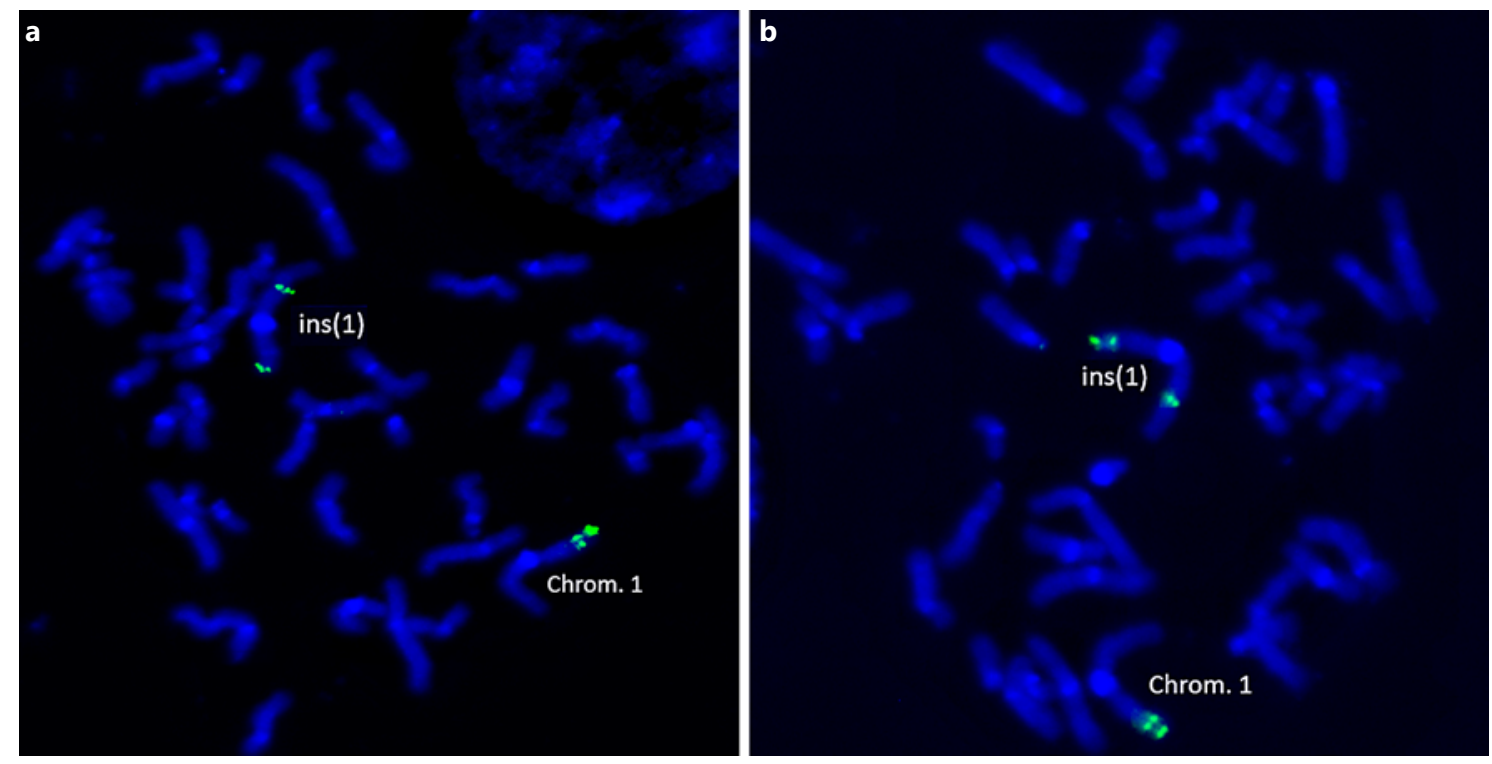

Fig. 5. FISH with an interstitial probe on 1q (RP11-239E10) and another on 1qter (both in green). a Metaphase from the mother showing one normal chromosome 1, with two signals on the long arm, and the other homologue with an insertion from the long arm to the short arm. b Metaphase from the proband showing one nor-

the short arm of chromosome 1 and in the long arm of chromosome 14, respectively, which underwent a balanced translocation with chromosome 18 ( Fig. 4). Combining the karyotype, CMA and FISH results, the mother's karyotype can be described as follows: 46,XX,ins(1) mal chromosome 1, with two signals on chromosome 1 , and a recombinant chromosome which has an additional segment inserted in the short arm. This result is in agreement with the duplication in 1q observed by chromosome microarray analyses. 


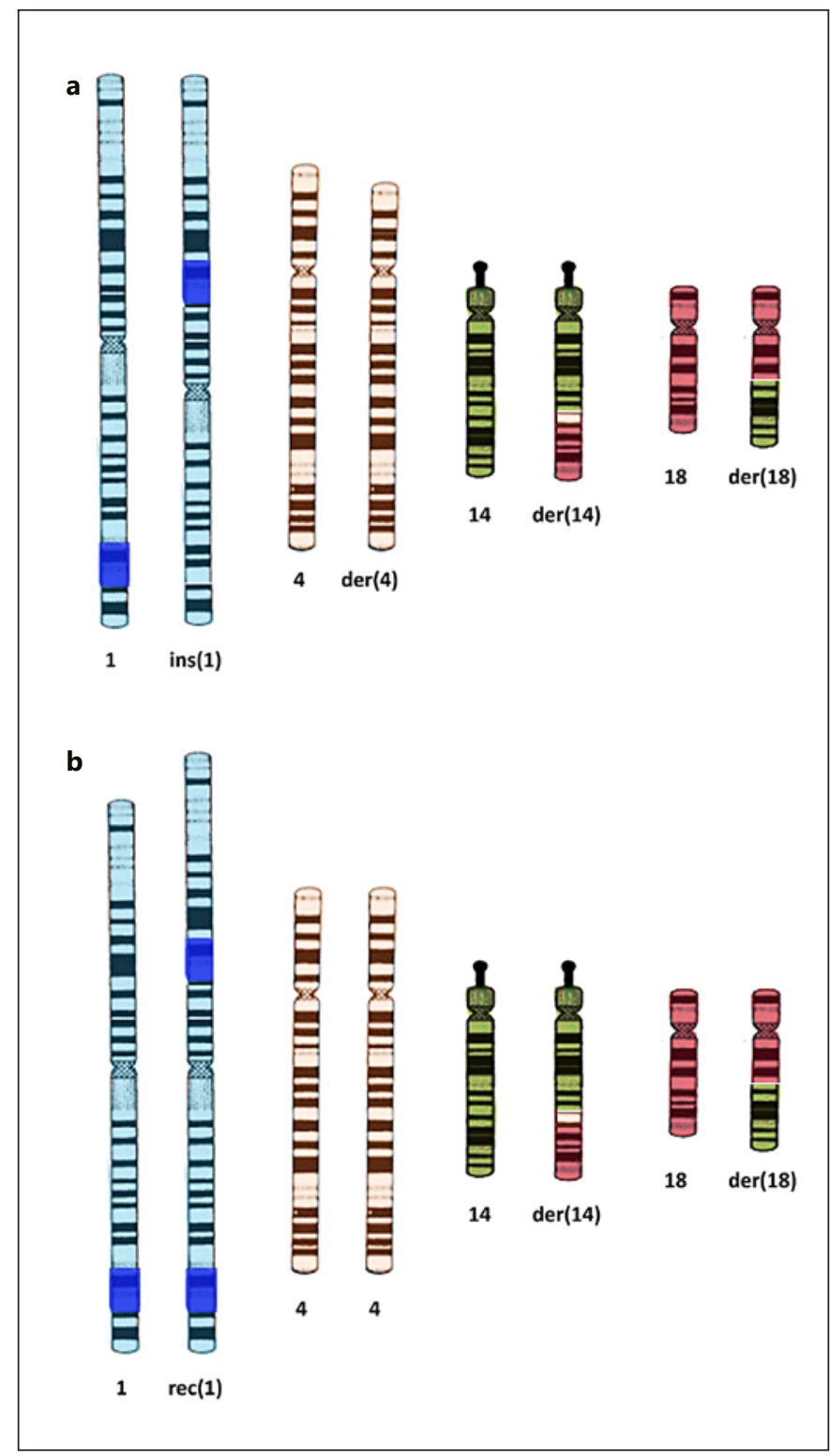

Fig. 6. Ideogram of the rearrangement of the mother (a) and the proband (b) showing normal chromosomes and their derivatives as interpreted after FISH analysis.

FISH also revealed that the son carried an unbalanced chromosome resulting from the recombination between chromosome 1 with an inserted segment from the long to the short arm and its normal homologue ( Fig. 5). FISH evaluation of the metaphases of the child also showed that he inherited the der(14) and its normal homologue, the der(18) and its normal homologue ( Fig. 3), and 2 normal chromosomes 4 ( Fig. 4). These results are in accordance with the extra material detected in the short arm of chromosome 4 and the long arm of chromosome 1 by CMA in the proband. The karyotype of the proband, based on CMA and FISH results, is described as follows: 46,XY,rec(1)(pter $\rightarrow$ p22.3:q41 $\rightarrow$ q43orq $43 \rightarrow q 41: p 22.3 \rightarrow$ qter)ins(1)(p22.3;q41q43)mat,der(14)(14pter $\rightarrow 14 \mathrm{q} 24.1:$ 4 p15.2 $\rightarrow$ 4p14or4p14 $\rightarrow 4$ p15.2:18q21.1 $\rightarrow 18 \mathrm{qter}) \operatorname{der}(18)$ $(18 \mathrm{pter} \rightarrow 18 \mathrm{q} 21.1: 14 \mathrm{q} 24.1 \rightarrow 14 \mathrm{qter}) \mathrm{t}(4 ; 14 ; 18)$ mat.

Figure 6 shows an ideogram of the normal and the rearranged chromosomes for a better visualization of the chromosomal break points.

\section{Discussion and Conclusion}

We described a patient with a malformation syndrome associated with an unbalanced CCR with at least 7 breaks and involving 4 chromosomes. The rearrangement resulted from segregation and recombination of the derivative chromosomes of the mother, a carrier of a balanced CCR. A search of the literature using the PubMed database and the keywords "duplication 4p" OR "trisomy 4p" OR "syndrome 4p" OR "duplication 1q" OR "trisomy 1q" OR "syndrome 1q" OR "complex chromosomal rearrangements", and a search of the Genome Browser database and the DECIPHER platform, found no case with the same set of alterations. However, recombinants with duplications or deletions arising from intra-chromosomal insertions as a result of a crossover in the interstitial or the inserted segment have been well documented for most chromosomes, including chromosome 1 [Madan and Menko, 1992; Jones et al., 2014; Silipgni et al., 2017]. The clinical features are summarized in Table 1.

Distal 1q duplication syndrome is characterized by the presence of intellectual disability/neurodevelopmental delay, a triangular face, downslanting and narrow palpebral fissures, a broad nasal bridge, a small pointed nose, micrognathia, low-set and posteriorly rotated ears with poorly formed helices, according to previously reported cases [Morris et al., 2016]. However, the clinical phenotype of trisomy 1q (q42qter) in cases involving another chromosome is not properly defined in the literature. Moreover, intrachromosomal insertions are rare phenomena, characterized by the presence of 3 breakpoints and the repositioning of a chromosome segment into another location in the same chromosome [Silipgni et al., 2017], that lead to imbalances, such as deletions or duplications, resulting from recombination within the inserted segment [Nowakowska et al., 2012].

Trisomy of the short arm of chromosome 4 was first described by Wilson et al. [1970]. Imbalance in the short arm of chromosome 4 leads to a variety of distinct clinical 
Table 1. Clinical findings in the present case and previously reported cases of duplication $4 \mathrm{p}$ and duplication $1 \mathrm{q}$

\begin{tabular}{|c|c|c|c|c|}
\hline & \multirow{2}{*}{$\begin{array}{l}\text { Our } \\
\text { patient }\end{array}$} & \multicolumn{2}{|l|}{ Duplication $4 \mathrm{p}$} & \multirow{2}{*}{$\begin{array}{l}\text { Duplication 1q } \\
\text { Morris et al., } \\
\text { [2016] - patient } 1\end{array}$} \\
\hline & & $\begin{array}{l}\text { Liang L et al., } \\
\text { [2016] }\end{array}$ & $\begin{array}{l}\text { Hemmat et al., } \\
\text { [2013] }\end{array}$ & \\
\hline Elongated/triangular face & + & + & + & + \\
\hline Epicanthus & - & + & ND & + \\
\hline Low-set or incompletely rotated ears & + & + & + & - \\
\hline Congenital heart disease & + & + & + & + \\
\hline Micrognathia & - & + & ND & ND \\
\hline Thin upper lip & + & + & + & + \\
\hline High-arched palate & - & ND & ND & + \\
\hline Jaw projection & - & ND & ND & + \\
\hline Facial hypoplasia & - & + & ND & ND \\
\hline Prominent nasal bridge & + & - & + & + \\
\hline Strabismus & + & + & ND & ND \\
\hline Microcephaly & + & + & + & - \\
\hline Intellectual disability & + & + & ND & + \\
\hline Motor delay & + & + & ND & ND \\
\hline Speech delay & + & + & ND & ND \\
\hline Short stature & + & + & + & + \\
\hline Limb defect & - & + & ND & ND \\
\hline Genu valgum & - & ND & ND & + \\
\hline Abnormalities of the genitourinary tract & + & + & + & ND \\
\hline Hypotonia & + & + & ND & ND \\
\hline Epilepsy & - & - & ND & ND \\
\hline Low birth weight & + & + & ND & + \\
\hline Low weight gain & + & + & + & + \\
\hline
\end{tabular}

ND, not described; +, present; -, absent.

symptoms such as intellectual disability, microcephaly, prenatal and postnatal growth restriction, and low-set ears [Gonzalez, 1977]. Therefore, clinical presentation of $4 \mathrm{p}$ duplication is considered nonspecific. The RBPJ gene (OMIM *147183) maps within the 4p15.2 region and was affected by the patient's duplication. $R B P J$ is known to be a transcription factor in the Notch/RBPJ signaling pathway and plays an important role in neurodevelopment [Liang L et al., 2016]. CCKAR (OMIM *118444) and CCKBR (OMIM*118445), receptors of cholecystokinin, are also included in the duplicated $4 \mathrm{p}$ segment. Both are expressed in the central and peripheral nervous system and show functional synergy in brain development. Cholecystokinin is a peptide hormone and one of the most abundant neuropeptides in the brain. It has been demonstrated to be a central regulator of neuronal circuits. It plays a vital role in the gastrointestinal system, satiation, memory, anxiety-related behaviors, and other neuropsychiatric disorders [Lee and Soltesz, 2011; Zwanzger et al., 2012; Nishimura et al., 2015; Washington et al., 2016]. Therefore, we hypothesize that duplication of the RBPJ and CCKAR genes may partly contribute to the neurodevelopmental anomalies in our patient.

Evaluation of CCRs and their potential phenotypic consequences is a common challenge in genetics. After an extensive review of cases of $1 \mathrm{q}$ and $4 \mathrm{p}$ duplications, it is hard to define which characteristics could be attributed to each of the duplication segments, considering the overlapping of some of the clinical findings for example, facial dysmorphisms (an elongated face, implanted low ears, a high palate), microcephaly, intrauterine growth restriction, intellectual disability, failure to thrive, and congenital heart disease.

The frequency of balanced CCR in the population may be underestimated, as they may not cause a phenotypic effect and the analyses used may not be able to detect them. To date, most of the breakpoints have been mapped using classical cytogenetic G-banding, FISH, whole chromosome painting FISH, M-FISH or SKY or multicolor banding [Zhang et al., 2009]. In cases of highly rearranged karyotypes, even the results of FISH analyses may be difficult to interpret. 
Whole-genome sequencing is superior to CMA in some respects as it can also detect balanced rearrangements with high resolution, resulting in higher detection accuracy. Moreover, the sequencing provides information on the genes disrupted by the translocations, which is unachievable using traditional cytogenetic methods, although the cost is much higher [Liang D et al., 2016], and the findings can be difficult to interpret. While it is clearly important to use the most advanced molecular techniques to discover small microduplications and/or microdeletions, microarrays are not suitable for detecting balance rearrangements and, as in our case, the use of Gbanding and FISH techniques were essential to be able to provide proper genetic counseling.

When an unbalanced translocation is identified in a patient, it is vital to test the parents for the presence of a balanced translocation to determine the recurrence risk in the family. It is important to highlight that the reproductive risks seem to be very specific for each carrier, and the precise prevalence is hard to establish. In our case, it was not only important to determine the presence of a balanced translocation in the mother, but was also important with respect to the patient's sister so that she will be able to receive the best genetic counseling when appropriate.

\section{Acknowledgement}

The authors would like to thank the family for their participation in this study.

\section{Statement of Ethics}

Written informed consent was obtained from the child's mother. Research was conducted ethically in accordance with the World Medical Association Declaration of Helsinki.

\section{Conflict of Interest Statement}

The authors have no conflicts of interest to declare.

\section{Funding Sources}

This research was supported by Instituto de Biociências (Universidade de São Paulo).

\section{Author Contributions}

A.E. Campos, E. Perrone, and C. Rosenberg substantially contributed to the conception and design of the study, the acquisition, analysis and interpretation of the data, and drafting or revising the article for important intellectual content. Clinical management and follow-up were undertaken by A. E. Campos, E. Perrone, and T. Vertemati. Karyotype, array and FISH analyses were carried out and performed by the Institute of Biosciences and GeneOne team. A.E. Campos, C. Rosenberg, M. França, S. Costa, A. Krepischi, A.C. Sousa, V. Lopes, L. Vieira, V. Nakano, M. Migliavacca, F. Milanezi, J. Silva, M. Cochak, P. Monfredini, A.C. Palumbo, J. Fernandes and E. Perrone were also involved in the analysis, discussion of the research, and revision of the manuscript.

\section{References}

Batista DA, Pai GS, Stetten G. Molecular analysis of a complex chromosomal rearrangement and a review of familial cases. Am J Med Genet. 1994;53:255-63.

Berend SA, Bodamer OA, Shapira SK, Shaffer LG, Bacino CA. Familial complex chromosomal rearrangement resulting in a recombinant chromosome. Am J Med Genet. 2002;109: $311-7$.

Ciccone R, Giorda R, Gregato G, Guerrini R, Giglio S, Carrozo R, et al. Reciprocal translocations: a trap for cytogenetists?. Hum Genet. 2005; 117:571-82.

De Gregori M, Ciccone R, Magini P, Pramparo T, Gimelli S, Messa J, et al. Cryptic deletions are a common finding in "balanced" reciprocal and complex chromosome rearrangements: a study of 59 patients. J Med Genet. 2007;44:750-62.

Gonzalez CH, Sommer A, Meisner LF, Elejalde BR, Opitz JM, Francke U. The trisomy 4p syndrome: case report and review. Am J Med Genet. 1977;1:137-56.

Hemmat M, Hemmat O, Anguiano A, Boyar FZ, El Naggar M, Wang JC, et al. Genotype-phe- notype analysis of recombinant chromosome 4 syndrome: an array-CGH study and literature review. Mol Cytogenet. 2013;6(1):17.

Jones CA, Kolomietz E, Maire G, Vlasschaert M, George AMJ, Reid DM, et al. PGD for a carrier of an intrachromosomal insertion using aCGH. Syst Biol Reprod Med. 2014;60(6):377-82.

Kurtas NE, Xumerlez L, Giussani U, Pansa A, Cardarelli L, Bertini V, et al. Insertional translocation involving an additional nonchromothriptic chromosome in constitutional chromothripsis: Rule or exception?. Mol Genet Genomic Med. 2019;7(2):e00496.

Lee SY, Soltesz I. Cholecystokinin: a multi-functional molecular switch of neuronal circuits. Dev Neurobiol. 2011;71:83-91.

Liang D, Wang Y, Ji X, Hu H, Zhang J, Meng L, et al. Clinical application of whole-genome lowcoverage next-generation sequencing to detect and characterize balanced chromosomal translocations. Clin Genet. 2016;91(4):605-10.

Liang L, Xie Y, Shen Y, Yin Q, Yuan H. A Rare de novo Interstitial Duplication at $4 \mathrm{p} 15.2$ in a Boy with Severe Congenital Heart Defects,
Limb Anomalies, Hypogonadism, and Global Developmental Delay. Cytogenet Genome Res. 2016;150:112-7.

Liao YP, Wang CJ, Liang M, Hu XM, Wu Q. Analysis of genetic characteristics and reproductive risks of balanced complex chromosome rearrangement carriers in China. Yi Chuan. 2017;39:396-412.

Madan K. Balanced complex chromosome rearrangements: Reproductive aspects. A review. Am J Med Genet A. 2012;158A:947-63.

Madan K, Menko FH. Intrachromosomal insertions: a case report and a review. Hum Genet. 1992;89(1):1-9.

Morris MLM, Baroneza JE, Teixeira P, Medina CTN, Cordoba MS, Versiani BR, et al. Partial 1q duplications and associated phenotype. Mol Syndromol. 2016;6:297-303.

Nazaryan L, Stefanou EG, Hansen C, Kosyakova $\mathrm{N}$, Bak M, Sharkey FH, et al. The strength of combined cytogenetic and mate-pair sequencing techniques illustrated by a germline chromothripsis rearrangement involving FOXP2. Eur J Hum Genet. 2014;22:338-43. 
Neira VA, Córdova-Fletes C, Grondin Y, Ramirez-Velazco A, Figuera LE, Ortíz-López $\mathrm{R}$, et al. Complex 9p rearrangement in an XY patient with ambiguous genitalia and features of both $9 \mathrm{p}$ duplication and deletion. Am J Med Genet A. 2012;158A(6):1498-502.

Nishimura S, Bilgüvar K, Ishigame K, Sestan N, Günel M, Louvi A. Functional synergy between cholecystokinin receptors CCKAR and CCKBR in mammalian brain development. PLoS One. 2015;10(4):e0124295.

Nowakowska BA, de Leeuw N, Ruivenkamp CA, Sikkema-Raddatz B, Crolla JA, Thoelen R, et al. Parental insertional balanced translocations are an important cause of apparently de novo CNVs in patients with developmental anomalies. Eur J Hum Genet. 2012;20:166-70.

Patsalis PC, Evangelidou P, Charalambous S, Sismani C. Fluorescence in situ hybridization characterization of apparently balanced translocation reveals cryptic complex chromosomal rearrangements with unexpected level of complexity. Eur J Hum Genet. 2004; 12:647-53.
Poot M, Haaf T. Mechanisms of Origin, Phenotypic Effects and Diagnostic Implications of Complex Chromosome Rearrangements. Mol Syndromol. 2015;6:110-34.

Rivera H, Domínguez MG. A familial rearrangement $(3 ; 5 ; 9)$ with paternal and maternal transmission leading to a duplication $3 \mathrm{p} /$ deletion 5p infant. Clinics (Sao Paulo). 2012;67: 669-72.

Scriven PN, Bint SM, Davies AF, Ogilvie CM. Meiotic outcomes of three-way translocations ascertained in cleavage-stage embryos: refinement of reproductive risks and implications for PGD. Eur J Hum Genet. 2014;22:748-53.

Shaffer LG, Lupski JR. Molecular Mechanisms for Constitutional Chromosomal Rearrangements in Humans. Annu Rev Genet. 2000;34: 297-329.

Silipgni R, Monfrini E, Baccarin M, Giangiobbe S, Lalatta F, Guerneri S, et al. Familial Duplication/Deletion of 1q42.13q43 as Meiotic Consequence of an Intrachromosomal Insertion in Chromosome 1. Cytogenet Genome Res. 2017;153(2):73-80.
Sismani C, Kitsiou-Tzeli S, Ioannides M, Christodoulou C, Anastasiadou V, Stylianidou G, et al. Cryptic genomic imbalances in patients with de novo or familial apparently balanced translocations and abnormal phenotype. $\mathrm{Mol}$ Cytogenet. 2008;1:15.

Washington MC, Williams K, Sayegh AI. The feeding responses evoked by endogenous cholecystokinin are regulated by different gastrointestinal sites. Horm Behav. 2016;78: 79-85.

Wilson MG, Towner JW, Coffin GS, Forsman I. Inherited pericentric inversion of chromosome no. 4. Am J Hum Genet. 1970;22:67990.

Zhang F, Carvalho CMB, Lupski JR. Complex human chromosomal and genomic rearrangements. Trends Genet. 2009;25:298-307.

Zwanzger P, Domschke K, Bradwejn J. Neuronal network of panic disorder: the role of the neuropeptide cholecystokinin. Depress Anxiety. 2012;29:762-74. 